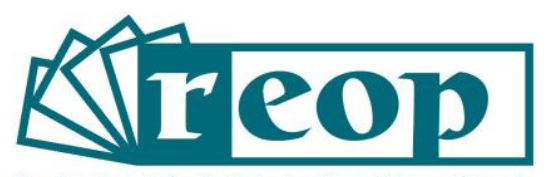

Revista Española de Orientación y Psicopedagogía

\title{
ANÁLISIS FACTORIAL, DE CONSISTENCIA INTERNA Y DE CONVERGENCIA DE LAS ESCALAS GRIT-O Y GRIT-S EN UNIVERSITARIOS ARGENTINOS. IMPLICACIONES PARA LA ORIENTACIÓN EN EDUCACIÓN SUPERIOR
}

\section{FACTORIAL ANALISYS, INTERNAL CONSISTENCY AND CONVERGENCE OF THE GRIT-O AND GRIT-S SCALES IN ARGENTINE UNIVERSITY STUDENTS. IMPLICATIONS FOR GUIDANCE IN HIGHER EDUCATION}

\author{
María Candela Tortul ${ }^{1}$ \\ Universidad Católica Argentina. Facultad "Teresa de Ávila". Centro de Investigación \\ Interdisciplinar en Valores, Integración y Desarrollo Social (CIIVIDS) - Paraná, Argentina \\ Universidad Austral - CONICET. Buenos Aires, Argentina \\ Florencia Teresita Daura \\ Universidad Austral. Escuela de Educación. Consejo Nacional de Investigaciones Científicas y \\ Técnicas (CONICET). Buenos Aires, Argentina \\ Belén Mesurado \\ Universidad Austral. Escuela de Educación. Consejo Nacional de Investigaciones Científicas y \\ Técnicas (CONICET). Buenos Aires, Argentina
}

\section{RESUMEN}

Se presentan dos estudios en los que se empleó un diseño instrumental en dos muestras de estudiantes universitarios de ambos sexos, de entre 17 y 30 años, que completaron la Escala Grit en su versión original (Duckworth et al., 2007) y abreviada (Duckworth y Quinn, 2009), y la adaptación española para estudiantes de la Utrecht-Work Engagement Scale (UWES-SS)

${ }^{1}$ Correspondencia: María Candela Tortul. Correo-e: candelatortul@uca.edu.ar /candelatortul@gmail.com 
(Schaufeli et al., 2002), adaptada y validada para su aplicación en población argentina (Mesurado et al., 2016). El Estudio 1 se realizó con una muestra de 233 alumnos para analizar la validez factorial de la adaptación argentina de las dos primeras escalas mencionadas, aplicando la matriz de correlaciones tetracóricas con el paquete estadístico Factor (Lorenzo-Seva y Ferrando, 2020) y el análisis de consistencia interna; asimismo, se analizó la convergencia de ambas escalas con la UWES-SS. Con la segunda muestra ( $n=235)$, se llevó a cabo el Estudio 2, en el que se realizó un análisis factorial confirmatorio de la Escala Grit-O y Grit-S. Los resultados son comparables con estudios similares efectuados con las escalas originales; y muestran que la Escala Grit, en sus dos versiones, posee sólidas propiedades psicométricas y buena validez de constructo, constituyéndose en instrumentos confiables para utilizarlos en la orientación psicopedagógica. Asimismo, la convergencia encontrada entre el Grit y el Engagement indica la importancia de estimular ambas capacidades de forma simultánea en población universitaria. Estos estudios brindan aportes de interés para el ámbito educativo en general y permiten profundizar el abordaje del Grit en el contexto mencionado.

Palabras clave: Grit; compromiso académico; escala; estudiantes universitarios; orientación psicopedagógica.

\section{ABSTRACT}

Two studies are presented in which an instrumental design was used with two samples of university students of both sexes, age range from 17 to 30 years, who completed the Grit Scale in its original version (Duckworth et al., 2007) and abbreviated (Duckworth \& Quinn, 2009), and the Spanish adaptation for students of the Ultrecht-Work Engagement Scale (UWES-SS) (Schaufeli et al., 2002), adapted and validated for its application in Argentine population (Mesurado et al., 2016). Study 1 was carried out with a sample of 233 students to analyze the factorial validity of the Argentine adaptation of the two Grit scales, applying the tetrachoric correlation matrix with the Factor statistical package (Lorenzo-Seva \& Ferrando, 2020) and the internal consistency analysis. Additionally, the convergence of both scales with the UWES-SS was analyzed. Study 2 was carried out with another sample $(\mathrm{N}=235)$ in which a confirmatory factor analysis of the Grit-O and Grit-S Scale was performed. The results are comparable with similar studies carried out with the original scales and show that the Grit Scale, in its two versions, has solid psychometric properties and good construct validity, becoming reliable instruments for psycho-pedagogical guidance. Likewise, the convergence found between Grit and Engagement indicates the importance of stimulating both abilities in the university population. These studies provide interesting contributions to the educational field in general and allow us to go deeper in the approach of Grit in the studied context.

Key Words: Grit; academic engagement; university students; psycho-pedagogical guidance.

\section{Cómo citar este artículo:}

Tortul, Ma .C., Daura, F.T. y Mesurado, B. (2020). Análisis factorial, de consistencia interna y de convergencia de las escalas Grit-O y Grit-S en universitarios argentinos. Implicaciones para la orientación en educación superior. Revista Española de Orientación y Psicopedagogía, 31(3), 109128. https://doi.org/10.5944/reop.vol.31.num.3.2020.29264 


\section{Introducción}

El término Grit es un neologismo en cuanto a su sentido y foco, que fue acuñado por Duckworth (2016), Duckworth et al. (2007) y se suele traducir como perseverancia o determinación.

Se trata de un rasgo de personalidad que se define como la perseverancia y la pasión para alcanzar metas a largo plazo y conlleva resistir con interés y esfuerzo los desafíos que se presentan en el proceso necesario para lograr los objetivos previamente fijados, independientemente de las dificultades, de la monotonía y de las vivencias de fracaso. De allí es que se la considere como la cualidad que tienen en común los grandes líderes (Duckworth et al., 2007). En efecto, a la persona perseverante se la reconoce por su capacidad de resistencia, ya que concibe la meta a alcanzar como una maratón y no como una carrera a toda velocidad (Duckworth, 2013, abril).

Del mismo modo, el Grit es un metaconstructo que posee una estructura jerárquica (Credé et al., 2017) al estar transversalizado por dos variables o dos factores denominados "consistencia de interés" y "perseverancia en el esfuerzo" (Duckworth, 2016; Duckworth et al., 2007) y que se refieren, respectivamente, a la tendencia a mantener los objetivos e intereses que se hayan propuesto y a trabajar intensamente ante los contratiempos y dificultades.

De acuerdo con Duckworth (2013, abril), esta fortaleza o rasgo de personalidad es independiente del nivel de cociente intelectual que se posee, ya que a través de numerosos estudios se ha mostrado que las personas que se destacan en lo académico, en su profesión o en otros ámbitos no lo hacen tanto por sus cualidades innatas, sino por la dedicación que emplean para alcanzar sus objetivos (Duckworth et al., 2007; Duckworth et al., 2009; Eskreis-Winkleret al., 2014; Roberton-Kraft y Duckworth, 2014).

Las investigaciones realizadas sobre el Grit muestran que este constructo posee un gran poder predictor sobre el rendimiento académico y profesional, sobre la permanencia en los estudios, en diversos ámbitos laborales y en la persistencia frente a tareas aburridas (Duckworth et al., 2010; Duckworth y Quinn, 2009; Duckworth et al., 2009; Eskreis-Winker et al., 2014; Perkins-Gough, 2013; Yeager et al., 2014).

Credé et al. (2017), al considerar la potencialidad del constructo, sostienen la importancia de profundizar a nivel teórico y empírico la vinculación que existe entre éste y otras variables con las que guarda una gran similitud y que en el campo de la psicología son estudiadas desde hace décadas. Entre ellas, particularmente, hacen referencia a la autoconciencia, la motivación de logro, diversos rasgos de personalidad (como la proactividad, el optimismo), el rendimiento, la capacidad intelectual, la satisfacción con la vida y diferencias sociodemográficas (sexo, edad, etc.).

Duckworth et al. (2007) diseñaron la escala Grit (Grit Scale, en su nombre original) que posee buenas propiedades psicométricas, ha sido validada en distintos contextos (Arco-Tirado et al., 2018; Becerra-Martín et al., 2016, septiembre) y resulta práctica para aplicarla, ya que se presenta en dos versiones: la escala Grit Original, conformada por 12 ítems (Grit-O), y la escala Grit Abreviada, formada por 8 ítems (Grit-S), que se distribuyen en los dos factores ya mencionados Consistencia de interés y Perseverancia en el Esfuerzo-; la cantidad de indicadores que conforman ambas formas del instrumento respetan las recomendaciones brindadas por la Comisión Internacional de Tests (International Tests Commission, 2001) para evaluar con precisión un constructo y evitar la saturación o el cansancio de las muestras que participen en un estudio. 
En la República Argentina, la escala aún no ha sido validada, por lo cual, en el presente trabajo se describen dos estudios con objetivos distintos: el estudio 1 se propone realizar el análisis Factorial (AF) de la adaptación argentina de la Escala Grit-O (Duckworth et al., 2007) y de la Escala Grit-S (Duckworth y Quinn, 2009), a fin de explorar su dimensionalidad y confirmar la estructura conceptual establecida con antelación por sus autores; el análisis de consistencia interna de ambos instrumentos y su convergencia con la escala UWES-SS.

El estudio 2 tiene por objeto detallar el Análisis Factorial Confirmatorio (AFC) de las dos versiones, a fin de confirmar que la estructura encontrada en ambas escalas puede obtenerse empíricamente.

Los datos recabados son de especial interés en el ámbito educativo en general y en el de la orientación psicopedagógica en particular, ya que muestran que los instrumentos son válidos para evaluar la perseverancia y la pasión que poseen los estudiantes para alcanzar objetivos más lejanos, como puede ser aprobar un curso académico, iniciar un nuevo nivel de enseñanza u obtener una titulación. Junto con ello, ofrecen la oportunidad de considerar estrategias de enseñanza que favorezcan el desarrollo de esta capacidad en el alumnado, sobre lo cual aún existe una laguna de conocimientos a cubrir.

\section{Estudio 1 \\ Método}

\section{Muestra}

La selección de la muestra se realizó mediante un procedimiento no probabilístico, intencional o de conveniencia. El tamaño de esta fue superior a 200 casos, superando el mínimo establecido para modelos estructurales simples (Tomarken y Waller, 2005).

La muestra estuvo compuesta por 233 estudiantes universitarios argentinos, que cursaban en su mayoría (88\%) $1^{\circ}$ y $2^{\circ}$ año de diferentes carreras de grado, a saber: el $42 \%$ Abogacía $(n=97)$, el $16 \%$ Lic. en Psicopedagogía $(n=36)$, el 12\% Lic. en Ciencias Políticas y Relaciones Internacionales $(n=28)$, el $11 \%$ Lic. en Psicología $(n=26)$, el $7 \%$ Ingeniería Civil $(n=17)$, el $6 \%$ Ingeniería Electrónica $(n=13)$, el $6 \%$ Contador Público $(n=13)$ y el $1 \%$ Lic. en Administración de Empresas $(n=2)$. El 13\% ( $n=30)$ estudiaba en 1 universidad pública y el $87 \%(n=203)$ en 2 universidades privadas. Con respecto a la procedencia, el $75 \%(n=175)$ era de la provincia de Entre Ríos y el 25\% $(n=58)$ de Buenos Aires. En relación con la distribución según sexo, el $65 \%$ eran mujeres $(n=151)$ y el $35 \%(n=82)$ varones. Por otra parte, el $57 \%(n=133)$ sólo estudiaba, mientras que el $43 \% \quad(n=100)$ restante trabajaba además de estudiar. Las edades de los estudiantes oscilaban entre los 17 y los 30 años, con una media de 20.30 (DT=2.44). 


\section{Instrumentos}

Los estudiantes universitarios que participaron en este estudio completaron la escala Grit-Original (Grit-O) de 12 ítems (Duckworth et al., 2007). La misma permite medir la perseverancia y la pasión por alcanzar metas a largo plazo y está conformada por dos dimensiones o factores: Consistencia de Interés (de aquí en más, $\mathrm{Cl}$ ) y Perseverancia en el Esfuerzo (en adelante, PE), compuestas por 6 ítems cada una. La modalidad de respuesta es en escala tipo Likert de 5 opciones (5 = Muy parecido a mí - 1 = En absoluto se parece a mí).

Junto con esta escala los alumnos completaron la versión Grit-S del instrumento (Short Grit Scale), la cual validaron Duckworth y Quinn en 2009. Este cuestionario contempla los mismos factores que la Escala Grit-O, aunque están compuestos por 4 ítems cada uno, dado que para su conformación se eliminaron los ítems 3 y 11 de la dimensión $\mathrm{Cl}$ y los ítems 1 y 10 para PE.

En su versión original, ambas presentaron adecuadas propiedades psicométricas. La Grit-O demostró una alta consistencia interna para la escala global (Alfa de Cronbach $=.85$ ) y para las subescalas: .84 para Cl y .78 para PE (Duckworth et al., 2007). Por su parte, la Grit-S ha demostrado una buena consistencia interna (.82 para la escala total, .77 para $\mathrm{Cl}$ y .70 para PE), estabilidad test-retest en las puntuaciones de la escala en el término de 12 meses $(r=.68)$ y validez convergente y discriminante (Duckworth y Quinn, 2009

Para estudiar la validez convergente, también se administró a los participantes la adaptación española para estudiantes de la Utrecht-Work Engagement Scale (UWES-SS) (Schaufeli et al., 2002; Schaufeli et al., 2002), adaptada y validada para su aplicación en población argentina (Mesurado et al., 2016).

Se trata de un instrumento de auto-reporte conformado por 14 ítems, que evalúa tres dimensiones del Compromiso Académico: vigor, dedicación y absorción; la primera de estas, el vigor (ítems 1 a 5), se refiere a la presencia de altos niveles de energía y resistencia mental durante el desarrollo de una tarea, como así también a la disposición a esforzarse y persistir en ella incluso ante dificultades; la dedicación (ítems 6 a 10) remite al sentido de importancia, entusiasmo, inspiración, orgullo y reto que la tarea le proporciona; y la tercera dimensión, la absorción (ítems 11 a 14), tiene que ver con el estado de concentración y felicidad que el sujeto experimenta mientras realiza la tarea; de allí es que, un alto nivel de Compromiso Académico se corresponde con altas puntuaciones en las tres dimensiones. La escala de respuesta es de tipo Likert, con 7 opciones que indican la frecuencia, que van de 0 (nunca) a 6 (siempre). Para calcular las dimensiones, se promedia el valor de los ítems correspondientes a las mismas, mientras que el valor de la escala total se obtiene promediando todos los ítems. Este instrumento presenta adecuados niveles de consistencia interna (alpha de Cronbach $=.88$ ) (Mesurado et al., 2016). El coeficiente de fiabilidad (alpha de Cronbach) obtenido en este estudio fue de .89 para la escala total, .83 de para vigor, .85 para dedicación y .76 para absorción.

\section{Procedimiento}

En el presente estudio se adoptó un diseño metodológico instrumental (Montero y León, 2007).

Se realizó una adaptación de la escala original de Duckworth et al. (2007) (Grit-O) a la lengua española (para la población argentina). Para ello se tuvieron en cuenta los pasos propuestos en la literatura sobre el tema (Muñiz et al., 2013): en primer lugar, se llevó a cabo una revisión sistemática del constructo Grit, luego se realizaron traducciones del inglés al español por dos personas calificadas para tal fin, y cada una de manera independiente propuso su versión de los 
ítems de la escala. A continuación, se realizó una traducción funcional del instrumento y se llevó a cabo una revisión de la adaptación por expertos en el área temática. Posteriormente, el equipo de investigación seleccionó, entre las diferentes opciones de traducción de los ítems, las que consideró más adecuadas. Y finalmente, se realizó una prueba piloto de la adaptación, que permitió identificar ciertos problemas de comprensión con algún ítem y realizar modificaciones. Por otra parte, tal como en la versión original, se mantuvo la modalidad de respuesta en escala tipo Likert de 5 opciones, pero se realizaron cambios en la redacción de las opciones de valoración a fin de que fueran más comprensibles para los estudiantes argentinos, quienes debían indicar en qué medida cada afirmación representaba su forma de ser, optando por una de las siguientes: 1. Nada, 2. Poco, 3. Más o menos, 4. Mucho o 5. Muchísimo.

Para analizar la versión abreviada (Grit-S), en este estudio se siguió el mismo procedimiento que emplearon Duckworth y Quinn (2009) cuando validaron la Grit-S (8 ítems): se suprimieron dos ítems por cada dimensión (específicamente se eliminaron los ítems 3 y 11 de la dimensión $\mathrm{Cl}$ y los ítems 1 y 10 para PE).

Las escalas fueron completadas por los estudiantes en contextos grupales (de entre 15 y 30 alumnos cada grupo), en días y horarios acordados con las autoridades de las instituciones educativas, quienes concedieron los permisos institucionales correspondientes. Los alumnos completaron las escalas en un tiempo aproximado de 10 minutos, ante la presencia del investigador, quien explicitó las instrucciones y se mostró disponible para responder cualquier consulta que surgiera. En la administración se tuvieron en cuenta las consideraciones éticas para este tipo de investigaciones: la participación fue voluntaria, lo cual quedó expresado en la firma del consentimiento informado por parte de cada estudiante de manera previa a la recolección de datos, luego de que se les explicaron los objetivos de la investigación y el tipo de participación solicitada; asimismo, se garantizó la confidencialidad de los datos recabados y su utilización exclusiva con fines investigativos.

Con respecto a los análisis estadísticos, a fin de determinar el poder discriminativo de los ítems y establecer si existen diferencias en las puntuaciones obtenidas para cada ítem entre sujetos con alto y bajo Grit, se efectuó una prueba t de Student para muestras independientes. Para estudiar la estructura empírica subyacente de la escala, tanto en su versión original (Grit-O) como en su versión reducida (Grit-S), se llevaron a cabo análisis factoriales exploratorios (AFE), aplicando la matriz de correlaciones tetracóricas, utilizando el paquete estadístico Factor (Lorenzo-Seva y Ferrando, 2020). Para analizar la consistencia interna de la escala se utilizó el Overall Reliability of fully Informative prior Oblique N-EAP scores (ORION) (Ferrando y LorenzoSeva, 2016). Finalmente, con el objetivo de estudiar la validez convergente del instrumento, se aplicó el análisis de correlación de Spearman para evaluar la asociación entre el Grit y el Compromiso Académico.

\section{Resultados}

Poder discriminativo de los ítems

Para evaluar el poder discriminativo de los ítems se procedió inicialmente a generar una agrupación visual con tres puntos de corte para la distribución de la variable de puntaje total de la Grit-O, generando cuatro grupos del $25 \%$ de la varianza cada uno. Para este análisis se tomaron 
dos grupos, uno ubicado en el primer cuartil (bajo Grit) conformado por 82 sujetos y otro ubicado en el tercer cuartil (alto Grit) conformado por 56 sujetos. Por medio de la prueba t de Student para muestras independientes, se compararon las medias de los dos grupos: Bajo Grit- y Alto Grit. Se encontró que los 12 ítems tienen una adecuada capacidad discriminativa ya que se obtuvieron diferencias estadísticamente significativas $(p=0.000)$ en la forma de responder entre los grupos de sujetos correspondientes al cuartil superior $\left(Q_{3}\right)$ y cuartil inferior $\left(Q_{1}\right)$ (ver Tabla 1$)$.

\section{Tabla 1}

Comparación de los valores medios y desvíos estándares entre los sujetos correspondientes al cuartil superior e inferior de Grit

\begin{tabular}{ccccccc}
\hline Ítems & \multicolumn{2}{c}{ Grupo Bajo Grit } & \multicolumn{2}{c}{ Grupo Alto Grit } & \multicolumn{2}{c}{$\begin{array}{c}\text { Valores } \\
\text { Estadísticos }\end{array}$} \\
\cline { 2 - 7 } & $\mathrm{M}$ & $\mathrm{DT}$ & $\mathrm{M}$ & $\mathrm{DT}$ & $t$ & $p$ \\
\hline $\mathbf{1}$ & 3.06 & .96 & 4.59 & .60 & -11.52 & 0.000 \\
\hline $\mathbf{2}$ & 2.59 & .92 & 3.80 & .96 & -7.52 & 0.000 \\
\hline $\mathbf{3}$ & 2.80 & 1.02 & 4.32 & .74 & -9.55 & 0.000 \\
\hline $\mathbf{4}$ & 3.13 & .98 & 4.45 & .83 & -8.20 & 0.000 \\
\hline $\mathbf{5}$ & 2.53 & 1.01 & 4.52 & .85 & -12.40 & 0.000 \\
\hline $\mathbf{6}$ & 3.20 & .87 & 4.52 & .60 & -10.57 & 0.000 \\
\hline $\mathbf{7}$ & 2.66 & .91 & 4.07 & .91 & -8.97 & 0.000 \\
\hline $\mathbf{8}$ & 2.48 & 1.06 & 4.50 & .81 & -12.73 & 0.000 \\
\hline $\mathbf{9}$ & 2.95 & .80 & 4.43 & .60 & -12.39 & 0.000 \\
\hline $\mathbf{1 0}$ & 2.23 & 1.09 & 4.22 & .92 & -11.50 & 0.000 \\
\hline $\mathbf{1 1}$ & 2.70 & .96 & 3.77 & 1.112 & -6.03 & 0.000 \\
\hline $\mathbf{1 2}$ & 3.22 & .77 & 4.80 & .444 & -15.28 & 0.000 \\
\hline
\end{tabular}

Fuente: Elaboración propia a partir de la salida del SPSS

Validez de constructo: Análisis Factorial Exploratorio

Para evaluar la validez factorial de la adaptación argentina de las escalas Grit-O y Grit-S, se llevó a cabo un AFE, aplicando la matriz de correlaciones tetracóricas, utilizando el paquete estadístico Factor (Lorenzo-Seva y Ferrando, 2020).

En lo que respecta a la escala Grit-O, de manera preliminar se evaluó el índice de adecuación muestral de Kaiser-Meyer-Olkin $(\mathrm{KMO}=.82)$ y la prueba de esfericidad de Bartlett $(x 2=903,86 ; p=$ .000 ), en base a los cuales se pudo establecer que era pertinente realizar el AFE. Mediante el análisis se confirmó la existencia de los dos factores propuestos por los autores para la escala original, explicando el 56,19\% de la varianza. El primer factor explicó el $40,16 \%$ de la varianza y el segundo explicó el 16,03\%. En la Tabla 2 pueden apreciarse las cargas factoriales de cada ítem 
de la adaptación argentina de la Escala Grit-O. Como se puede observar, en Consistencia de Interés saturaron los ítems: 2, 3, 5, 7, 8 y 11 y en Perseverancia en el Esfuerzo saturaron los ítems: 1,4, 6, 9, 10 y 12 .

Por su parte, en cuanto a la escala Grit-S, también resultó pertinente realizar el AFE, $(\mathrm{KMO}=.79$; prueba de esfericidad de Bartlett: $\chi 2=575,56 ; p=.000)$, que evidenció dos factores, cuyo porcentaje de varianza explicado fue superior, en comparación con el anterior análisis: $64,42 \%$. El primer factor explicó el $47,06 \%$ de la varianza y el segundo explicó el $17,37 \%$. En este caso, los ítems que saturaron en Consistencia de Interés fueron: 2, 5, 7 y 8 y en Perseverancia en el Esfuerzo saturaron los ítems: 4, 6, 9 y 12. Las cargas factoriales de cada ítem de la adaptación argentina de la escala Grit-S también pueden observarse en la Tabla 2.

\section{Tabla 2}

Estructura factorial de la adaptación argentina de la escala Grit-O y de la Grit-S

\begin{tabular}{|c|c|c|c|c|}
\hline \multirow{2}{*}{ Ítems } & \multicolumn{2}{|c|}{ GRIT-O } & \multicolumn{2}{|c|}{ GRIT-S } \\
\hline & $\begin{array}{c}\text { C. } \\
\text { Interés }\end{array}$ & $\begin{array}{c}\text { P. } \\
\text { Esfuerzo }\end{array}$ & $\begin{array}{c}\text { C. } \\
\text { Interés }\end{array}$ & $\begin{array}{c}\text { P. } \\
\text { Esfuerzo }\end{array}$ \\
\hline $\begin{array}{l}\text { 1- He superado adversidades para lograr un desafío } \\
\text { importante. }\end{array}$ & -0.41 & .678 & - & - \\
\hline $\begin{array}{l}\text { 2- Las ideas y proyectos nuevos a veces me distraen de } \\
\text { los anteriores. }{ }^{*}\end{array}$ & .690 & .016 & .703 & -.030 \\
\hline 3- Mis intereses cambian de año en año*. & .579 & .157 & - & - \\
\hline $\begin{array}{l}\text { 4- Los contratiempos y dificultades no me desaniman. } \\
\text { No me doy por vencido fácilmente. }\end{array}$ & .030 & .578 & -.016 & .656 \\
\hline $\begin{array}{l}\text { 5- Me obsesiono con ciertas ideas o proyectos por poco } \\
\text { tiempo, pero luego pierdo el interés }{ }^{*} \text {. }\end{array}$ & .701 & .235 & .751 & .184 \\
\hline 6- Soy una persona muy trabajadora & .018 & .703 & .003 & .725 \\
\hline $\begin{array}{l}\text { 7- Con frecuencia me fijo una meta, pero después elijo } \\
\text { seguir otra*. }\end{array}$ & .542 & .215 & .559 & .292 \\
\hline $\begin{array}{l}\text { 8- Me cuesta mantener mi atención en proyectos que } \\
\text { tardan muchos meses en concretarse }{ }^{\star} \text {. }\end{array}$ & .542 & .297 & .588 & .269 \\
\hline 9- Termino todo lo que empiezo. & .277 & .530 & .251 & .541 \\
\hline $\begin{array}{l}\text { 10- He logrado una meta u objetivo que me llevó años } \\
\text { de trabajo. }\end{array}$ & .006 & .655 & - & - \\
\hline $\begin{array}{l}\text { 11- Me intereso por nuevas actividades cada pocos } \\
\text { meses*. }^{*}\end{array}$ & .623 & -.058 & - & - \\
\hline 12- Soy perseverante. Nunca me rindo. & .063 & .822 & .035 & 847 \\
\hline
\end{tabular}

* Ítems de puntuación invertida

Fuente: Elaboración propia a partir de la salida del Factor.

En la Tabla 3 siguiente se muestran los valores de comunalidad alcanzados por cada ítem en ambas versiones de la escala Grit, a los cuales se les otorgó distinto nivel de significación (óptima, a aquellos ítems cuyas comunalidades superan los 0,70 puntos; moderada, a los ítems cuyas comunalidades se encuentran se encuentran entre 0,40 y 0,70 puntos; o mínima, a los ítems que poseen una comunalidad baja, cercana a 0,30 puntos) de acuerdo con las recomendaciones teóricas existentes (Lloret-Segura et al., 2014). Puede observarse que en la Grit-O, casi todos los ítems poseen una condición moderada, con excepción del ítem 11 y del 4, que alcanzó la menor puntuación, mientras que el ítem 12 puede considerarse dentro de la categoría óptima. Por su parte, en la Grit-S, ningún ítem obtuvo comunalidad mínima, casi todos los ítems poseen una condición moderada y el ítem 12, como en la otra versión, tiene la condición óptima. 


\section{Tabla 3}

Significación de las comunalidades alcanzadas por cada uno de los ítems que integran las escalas Grit-O y Grit S

\begin{tabular}{|c|c|c|c|c|c|c|}
\hline \multirow{2}{*}{ Ítems } & \multicolumn{3}{|c|}{ GRIT O } & \multicolumn{3}{|c|}{ GRIT S } \\
\hline & Óptima & Moderada & Mínima & Óptima & Moderada & Mínima \\
\hline 1 & & 0.447 & & & & \\
\hline 2 & & 0.482 & & & 0.482 & \\
\hline 3 & & 0.408 & & & & \\
\hline 4 & & & 0.355 & & 0.423 & \\
\hline 5 & & 0.635 & & & 0.689 & \\
\hline 6 & & 0.514 & & & 0.527 & \\
\hline 7 & & 0.402 & & & 0.420 & \\
\hline 8 & & 0.532 & & & 0.522 & \\
\hline 9 & & 0.436 & & & 0.445 & \\
\hline 10 & & 0.431 & & & & \\
\hline 11 & & & 0.372 & & & \\
\hline 12 & 0.707 & & & 0.739 & & \\
\hline
\end{tabular}

Fuente: elaboración propia a partir de la salida del Factor.

\section{Consistencia Interna}

Para analizar la consistencia interna, se estimó la confiabilidad mediante ORION (acrónimo de "Overall Reliability of fully Informative prior Oblique N-EAP scores") (Ferrando y Lorenzo-Seva, 2016). En la escala Grit-O (12 ítems), el valor hallado para el factor Cl (6 ítems) resultó de 89 y para el factor PE, (6 ítems) fue de .92. En cuanto a la escala Grit -S (8 ítems), se halló un valor de .87 para el factor $\mathrm{Cl}$ (4 ítems) y de .89 para $P E$ (4 ítems). Los resultados obtenidos evidencian una buena consistencia interna en ambas versiones y puede observarse que la escala Grit-O obtuvo mejores coeficientes, en comparación con la Grit-S.

\section{Validez convergente: Relación entre Grit y Compromiso Académico}

Para evaluar la validez convergente de la adaptación argentina de la escala Grit-O y de la escala Grit-S, se realizaron correlaciones Rho de Spearman con la Utrecht-Work Engagement Scale (UWES-SS). El factor $\mathrm{Cl}$ presentó correlaciones positivas muy significativas desde el punto de vista estadístico con todas las dimensiones del Compromiso Académico: Vigor (Rho=.16; $p=.00$ ), Dedicación (Rho=.23; $p=.00$ ) y Absorción (Rho $=.18 ; p=.00$ ). Por su parte, el factor $P E$ también evidenció correlaciones positivas muy significativas con las tres dimensiones del Compromiso: Vigor (Rho=.15; $p=.00)$, Dedicación (Rho=.49; $p=.00$ ) y Absorción (Rho=.36; $p=.00)$.

Al analizar la escala Grit-S, se encontraron asociaciones significativas con las mismas variables del Compromiso Académico y los valores de dichas correlaciones resultaron similares. El factor $\mathrm{Cl}$ presentó correlaciones positivas muy significativas desde el punto de vista estadístico con todas las dimensiones del Compromiso Académico: Vigor (Rho=.15; $p=.00$ ), Dedicación (Rho $=.27 ; p=.00)$ y Absorción (Rho=.18; $p=.00$ ). Por su parte, el factor $P E$ también evidenció correlaciones positivas muy significativas con las tres dimensiones del Compromiso: Vigor (Rho=.18; $p=.00$ ), Dedicación (Rho=.44; $p=.00$ ) y Absorción (Rho=.36; $p=.00$ ) (Tabla 4). 


\section{Tabla 4}

Correlaciones entre los factores de la adaptación argentina de la escala Grit-O y Grit-S y las dimensiones del Utrecht-Work Engagement Scale (UWES-SS)

\begin{tabular}{|c|c|c|c|c|c|c|c|}
\hline \multicolumn{2}{|c|}{ Variables del UWES-SS } & \multicolumn{2}{|c|}{ Vigor } & \multicolumn{2}{|c|}{ Dedicación } & \multicolumn{2}{|c|}{ Absorción } \\
\hline & & Grit-O & Grit-S & Grit-O & Grit-S & Grit-O & Grit-S \\
\hline \multirow[t]{2}{*}{$\begin{array}{l}\text { Variables de las } \\
\text { escalas Grit }\end{array}$} & $\begin{array}{l}\text { Consistencia de } \\
\text { Interés }\end{array}$ & $.161^{\star *}$ & $.148^{\star *}$ & $.231^{\star *}$ & $.266^{\star *}$ & $.181^{* *}$ & $.177^{* *}$ \\
\hline & $\begin{array}{l}\text { Perseverancia } \\
\text { en el Esfuerzo }\end{array}$ & $.152^{\star \star}$ & $.176^{\star *}$ & $.494^{\star *}$ & $.440^{* *}$ & $.360^{\star *}$ & $.365^{\star *}$ \\
\hline
\end{tabular}

\section{Estudio 2}

Método

\section{Muestra}

La muestra del estudio 2 estuvo compuesta por 235 estudiantes universitarios argentinos, distintos de los incluidos en el estudio 1 , quienes en su mayoría (86\%) cursaban $1^{\circ}$ y $2^{\circ}$ año de diferentes carreras de grado, entre ellas: el 38\% Abogacía $(n=90)$, el $17 \%$ Lic. en Psicopedagogía $(n=39)$, el $14 \%$ Lic. en Ciencias Políticas y Relaciones Internacionales $(n=32)$, el $11 \%$ Lic. en Psicología $(n=25)$, el $8 \%$ Ingeniería Civil $(n=19)$, el 6\% Contador Público $(n=13)$, el $5 \%$ Ingeniería Electrónica $(n=11)$ el $1 \%$ Lic. en Administración de Empresas $(n=3)$ y el $1 \%$ Ingeniería Electromecánica $(n=2)$. El $14 \%(n=32)$ estudiaba en 1 universidad pública y el $86 \%(n=203)$ en 2 universidades privadas. Con respecto a la procedencia, el $75 \%(n=176)$ era de la provincia de Entre Ríos y el $25 \%(n=59)$ de la provincia de Buenos Aires. En relación a la distribución según sexo, el $60 \%$ eran mujeres $(n=142)$ y el $40 \%(n=93)$ varones. Por otra parte, el $55 \%(n=129)$ sólo estudiaba, mientras que el $45 \% \quad(n=106)$ restante trabajaba además de estudiar. Las edades de los estudiantes se encontraban entre los 17 y los 30 años, con una media de 20.57 (DT=2.85).

La selección de la muestra se realizó mediante un procedimiento no probabilístico, intencional o de conveniencia. El tamaño de la misma fue superior a 200 casos, superando el mínimo establecido para modelos estructurales simples (Tomarken y Waller, 2005).

\section{Instrumentos}

Tal como se describió en el estudio 1, se administró a los participantes la adaptación de la escala Grit-Original (Grit-O) de 12 ítems (Duckworth et al., 2007), la versión Grit-S del instrumento (Short Grit Scale) (Duckworth y Quinn, 2009) y la adaptación española para estudiantes de la Utrecht- 
Work Engagement Scale (UWES-SS), desarrollada por Schaufeli et al. (2002), adaptada y validada para su aplicación en población argentina (Mesurado et al., 2016).

\section{Procedimiento}

Para confirmar la estructura bifactorial de la adaptación argentina de la escala Grit, en sus dos versiones, original y abreviada, se llevaron a cabo análisis factoriales confirmatorios (AFC), utilizando el paquete estadístico AMOS .22. Para estudiar la consistencia interna se utilizó el Alfa de Cronbach y se calculó la varianza extraída media (Average Variance Extrated - AVE) y la fiabilidad compuesta del constructo. Finalmente, para estudiar la validez convergente se estudió la relación entre los dos factores que componen la escala y las dimensiones de Compromiso Académico.

\section{Resultados}

Con el objetivo de poner a prueba el modelo de dos dimensiones de la adaptación argentina de la escala Grit-O y de la Grit-S surgido del AFE del estudio 1, se llevaron a cabo análisis factoriales confirmatorios (AFC) utilizándose como método de estimación de parámetros el de Máxima Verosimilitud. Para evaluar el ajuste de los modelos se tuvieron en cuenta: el índice de bondad de ajuste (GFI), el índice ajustado de bondad de ajuste (AGFI), el índice de ajuste incremental (IFI) y el índice comparativo de ajuste (CFI). Se calcularon como medidas del error el Root Mean Square Residual (RMR) y el promedio de los residuales estandarizados al cuadrado (RMSEA).

En el caso de la Grit-O, los resultados del ajuste del modelo fueron los siguientes: $x 2(53)=$ $171.40, \mathrm{p}=.000, \mathrm{X} 2 / \mathrm{gl}=3.23, \mathrm{GFI}=.91, \mathrm{AGFI}=.87, \mathrm{IFI}=.89, \mathrm{CFI}=.89, \mathrm{RMR}=.070$ y RMSEA= .089. Por su parte, para la Grit-S se hallaron los siguientes resultados: $X 2(19)=59.81, p=.000$, $\mathrm{X} 2 / \mathrm{gl}=3.15, \mathrm{GFI}=.94, \mathrm{AGFI}=.88, \mathrm{IFI}=.88, \mathrm{CFI}=.88, \mathrm{RMR}=.076$ y $\mathrm{RMSEA}=.096$.

En las Figuras 1 y 2 pueden observarse los modelos de medición y las cargas factoriales estandarizadas de las adaptaciones de las escalas Grit-O y Grit-S, respectivamente. 


\section{Figura 1}

Modelo de medición y cargas factoriales estandarizadas de la adaptación argentina de la escala Grit-O

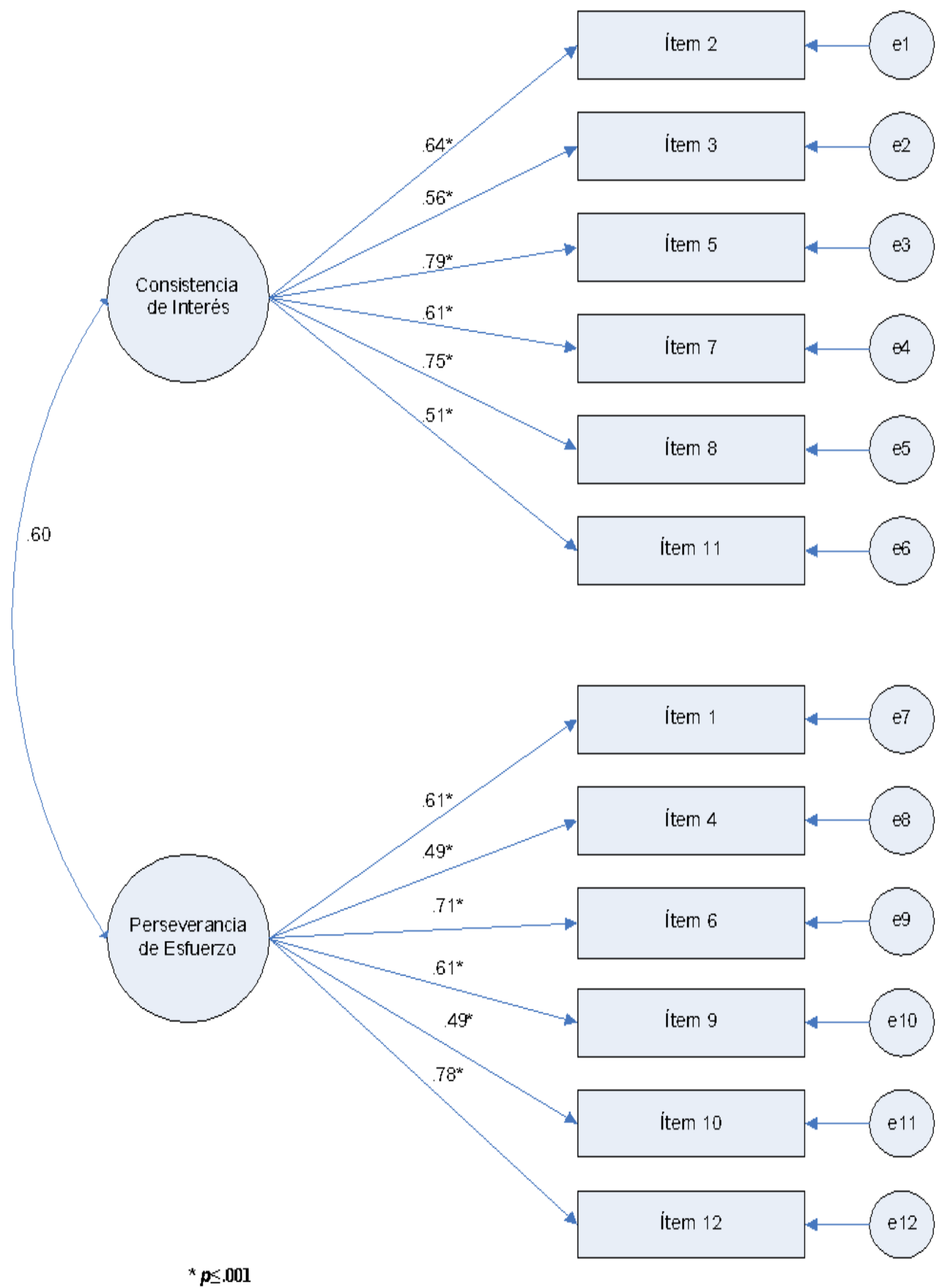

Fuente: elaboración propia a partir de la salida del AMOS 


\section{Figura 2}

Modelo de medición y cargas factoriales estandarizadas de la adaptación argentina de la escala Grit-S

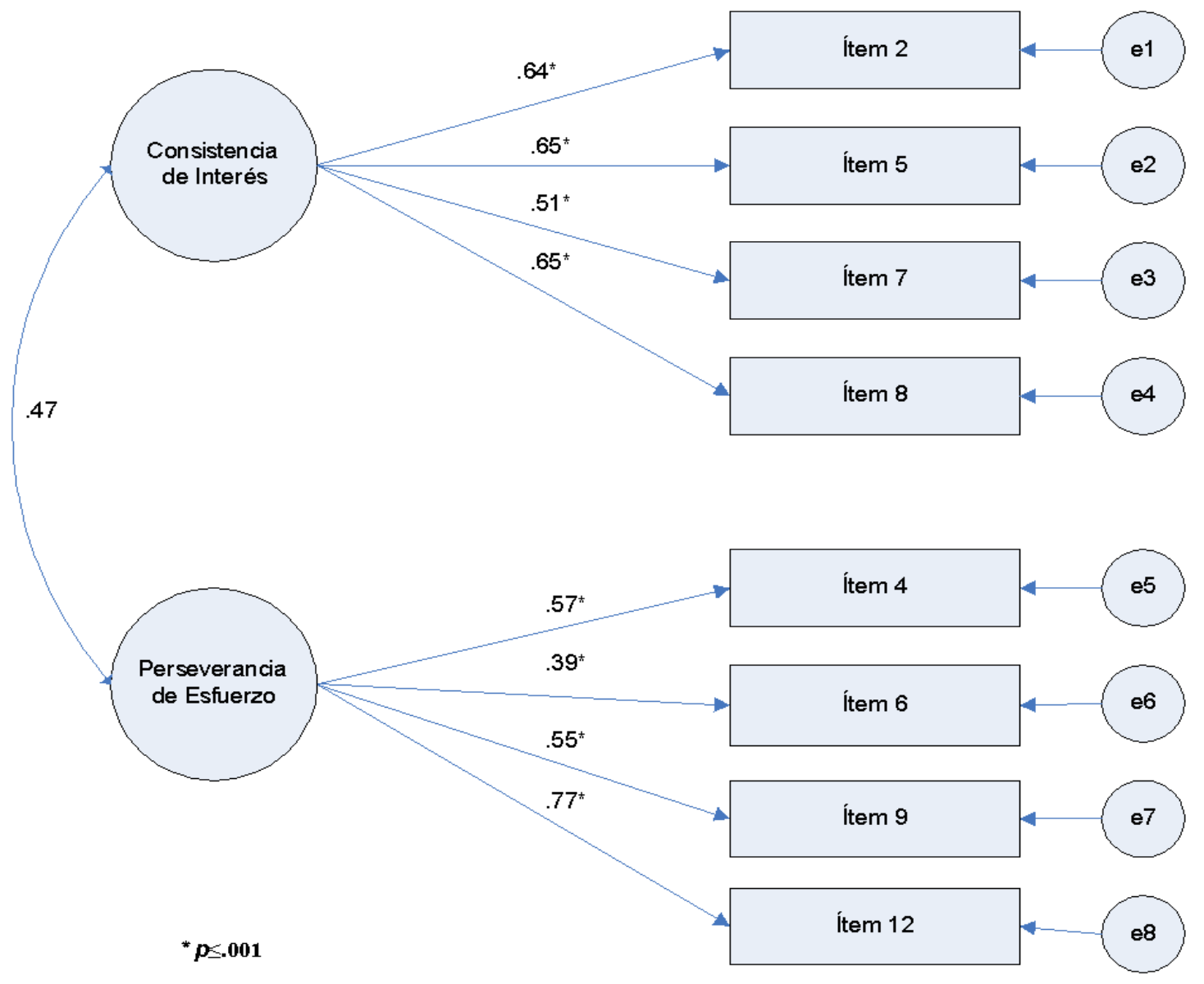

Fuente: elaboración propia a partir de la salida del AMOS

\section{Consistencia Interna}

Luego del AFC, se llevó a cabo el análisis de consistencia interna. En relación con la escala GritO, el Alfa de Cronbach para $\mathrm{Cl}$ (6 ítems), fue de .77, para PE (6 ítems), fue de de .73, mientras que para la totalidad de la escala (12 ítems) fue de .76; los valores del AVE fueron de .42 para Cl, de .39 para PE y de .40 para la escala total y la fiabilidad compuesta del constructo fue de .56 para $\mathrm{Cl}$, de .57 para $\mathrm{PE}$ y de .72 para la escala total.

En cuanto a la escala Grit-S (8 ítems), el Alfa de Cronbach fue de .73 para la escala total, de .76 para $\mathrm{Cl}$ (4 ítems), y de .73 para PE (4 ítems); los valores del AVE fueron de .38 para $\mathrm{Cl}$, de .34 para PE y de .36 para la escala total y la fiabilidad compuesta del constructo fue de .45 para $\mathrm{Cl}$, de .40 para PE y de .60 para la escala total. 


\section{Validez convergente: Relación entre Grit y Compromiso Académico}

Con el fin de estudiar la validez convergente de la adaptación argentina de la escala Grit, se evaluó su asociación con la Utrecht-Work Engagement Scale (UWES-SS), mediante un análisis de correlación de Spearman. El factor $\mathrm{Cl}$ presentó correlaciones positivas muy significativas con dos dimensiones del Compromiso Académico: Vigor $(R h o=.20 ; p=.00)$ y Absorción $R h o=.20 ; p=.00)$ y una asociación significativa con Dedicación $(R h o=.19 ; \mathrm{p}=.05)$. Por su parte, el factor PE evidenció correlaciones muy significativas positivas con: Dedicación (Rho=.42; $\mathrm{p}=.00$ ) y Absorción (Rho=.36; $\mathrm{p}=.00)$.

Al analizar la escala Grit-S, se encontraron asociaciones significativas con las mismas variables del Compromiso Académico y los valores de dichas correlaciones resultaron similares. EI factor $\mathrm{Cl}$ presentó correlaciones positivas muy significativas con las 3 dimensiones del Compromiso Académico: Vigor (Rho=.18; $\mathrm{p}=.00$ ), Dedicación $(\mathrm{Rho}=.26 ; \mathrm{p}=.00$ ) y Absorción $(\mathrm{Rho}=.25 ; \mathrm{p}=.00)$. Por su parte, el factor $\mathrm{PE}$ evidenció una correlación positiva con Vigor (Rho= $.14 ; p=.05)$ y correlaciones muy significativas positivas con: Dedicación (Rho=.39; $p=.00)$ y Absorción (Rho=.37; $\mathrm{p}=.00$ ) (Tabla 5).

\section{Tabla 5}

Correlaciones entre los factores de la adaptación argentina de la escala Grit-O y Grit-S y las dimensiones de la Utrecht-Work Engagement Scale (UWES-SS)

\begin{tabular}{|c|c|c|c|c|c|c|c|}
\hline \multicolumn{2}{|c|}{ Variables del UWES-SS } & \multicolumn{2}{|c|}{ Vigor } & \multicolumn{2}{|c|}{ Dedicación } & \multicolumn{2}{|c|}{ Absorción } \\
\hline & & Grit-O & Grit-S & Grit-O & Grit-S & Grit-O & Grit-S \\
\hline \multirow[t]{2}{*}{$\begin{array}{l}\text { Variables de las } \\
\text { escalas Grit }\end{array}$} & $\begin{array}{l}\text { Consistencia de } \\
\text { Interés }\end{array}$ & $.196^{\star *}$ & $.185^{\star *}$ & $.190^{*}$ & $.264^{\star *}$ & $.205^{\star *}$ & $.253^{* *}$ \\
\hline & $\begin{array}{l}\text { Perseverancia } \\
\text { en el Esfuerzo }\end{array}$ & .125 & $.145^{\star}$ & $.416^{\star \star}$ & $.390^{* *}$ & $.358^{* *}$ & $.370^{\star *}$ \\
\hline
\end{tabular}

\section{Discusión}

La finalidad de esta investigación consistió en realizar el análisis factorial, de consistencia interna y de convergencia de las escalas Grit Original (Duckworth et al., 2007) y Grit abreviada (Duckworth y Quinn, 2009) en estudiantes universitarios argentinos. En relación con el objetivo del estudio 1, puede afirmarse que los ítems de la escala Grit discriminan adecuadamente, es decir, que los reactivos distinguen correctamente entre quienes poseen el atributo y quienes no. Asimismo, los resultados hallados en el AFE confirman la existencia de los dos factores propuestos por los autores para la escala original: $\mathrm{Cl}$ y $\mathrm{PE}$, explicando un porcentaje adecuado de la varianza. Ambos factores también se evidenciaron en la versión breve, aunque en este caso fue mayor la proporción de la varianza explicada por los mismos. La distribución de los ítems entre factores coincide con los hallados por los autores de la escala y por otros estudios realizados en diferentes culturas (Akin et al., 2011, septiembre; Barriopedro et al., 2018; Fernández Martín et al., 
2018; Nishikawa et al., 2015). Asimismo, al analizar los valores de comunalidad alcanzados por cada ítem en ambas versiones de la escala Grit, se pudo observar que en la Grit-O, casi todos los ítems poseen una significación moderada, con excepción de los ítems 11 y 4, que presentaron una comunalidad mínima, mientras que el ítem 12 presentó una significación óptima. Por su parte, en la Grit-S, en la cual se eliminan los ítems 3 y 11 del factor $\mathrm{Cl}$ y los ítems 1 y 10 correspondientes al factor PE, ningún ítem obtuvo una comunalidad mínima y todos los ítems, con excepción del 12 que alcanzó una comunalidad óptima, poseen una condición moderada. Al respecto, es importante tener en cuenta que Duckworth et al. (2007), en su investigación, encontraron que ninguno de los dos factores resulta más predictivo que el otro y afirman que juntos poseen un mayor poder de predicción que cada uno por separado, por lo cual recomiendan emplear el puntaje total de la escala como medida del Grit.

Con respecto a la fiabilidad de los instrumentos, puede afirmarse que se hallaron niveles satisfactorios de consistencia interna en las subescalas de ambas versiones ( $\mathrm{Cl}$ y $\mathrm{PE}$ ), con valores que oscilaban entre .87 y .92 y que los índices de fiabilidad resultaron mejores en la escala Grit-O que en la Grit-S. Estos resultados muestran, por un lado, que el instrumento mide lo que dice medir, que los ítems que lo integran evalúan el mismo constructo y que están altamente correlacionados; por el otro, son similares a los informados en estudios anteriores (Barriopedro et al., 2018; Duckworth et al., 2007; Duckworth y Quinn, 2009; Fernández Martín et al., 2018).

Paralelamente, teniendo en cuenta los resultados de numerosas investigaciones previas que hallaron una asociación significativa positiva entre el Grit y el Compromiso o Engagement (Datu et al., 2016; Hodge et al., 2018; Suzuki et al., 2015; Von Culin et al., 2014), este trabajo también se propuso estudiar la validez constructiva convergente de la escala Grit en sus dos versiones analizando su correlación con la escala de Compromiso Académico. En el estudio 1, tanto en la escala Grit-O como en la Grit S, los factores $\mathrm{Cl}$ y PE presentaron correlaciones muy significativas positivas con las tres dimensiones del Compromiso: Vigor, Dedicación y Absorción y se obtuvieron resultados similares en el estudio 2. Estos resultados, aportan sustento empírico a la relación que se esperaba teóricamente y permiten ver que, a mayores niveles de PE y de $\mathrm{Cl}$, se presentan en los estudiantes universitarios mayores niveles de resistencia mental (vigor), de entusiasmo e inspiración (dedicación) y de concentración y felicidad para realizar una tarea, lo que concuerda con los hallazgos de las investigaciones previamente mencionadas y con lo afirmado por Duckworth (2013, abril) con respecto a que, las personas con mayores niveles de Grit, conciben las metas que se proponen como una maratón y no como una carrera, es decir, equilibran sus esfuerzos y continúan actuando con toda la dedicación, el entusiasmo y el interés necesarios para lograr los objetivos que se proponen, sin rendirse ante los obstáculos que se le presenten.

El estudio 2 se desarrolló con el objetivo de confirmar la estructura factorial de la adaptación argentina de la escala Grit-O y de la Grit-S, los resultados del AFC, tanto en la versión original como en la versión breve, indicaron un ajuste pobre entre el modelo teórico propuesto y los datos empíricos. Si bien el valor relativo de chi cuadrado (x2/gl) presentó valores cercanos a 3 en ambas escalas, los cuales son acordes con los valores propuestos por la literatura como aceptables y que se encuentran entre 2 y 5 puntos (Bentler, 1990) y el índice de bondad de ajuste (GFI) presentó valores superiores a .90; los demás indicadores analizados (el índice comparativo de ajuste (CFI), el índice ajustado de bondad de ajuste (AGFI) y el índice de ajuste incremental (IFI), que comparan el modelo propuesto con un modelo de independencia entre las variables consideradas, presentaron valores cercanos pero inferiores a .90 , siendo éste el límite inferior aceptable propuesto en la literatura (Hu y Bentler, 1998; Kline, 2005). Por otra parte, de manera similar a los resultados hallados para la versión original por parte de Duckworth et al. (2007), las medidas del error RMR y RMSEA no pueden considerarse satisfactorias (Hu y Bentler, 1998).

De esta manera, los resultados del análisis factorial confirmatorio, evidencian en la escala Grit-O un ajuste pobre del modelo de dos factores y son congruentes con los informados por los autores del instrumento (Duckworth et al., 2007) que tampoco hallaron un buen ajuste para su modelo $(\mathrm{CFI}=.83, \mathrm{RMSEA}=.11)$ y por otras investigaciones (Barriopedro et al., 2018). Asimismo, en lo que respecta a la escala Grit-S, en el presente trabajo, a diferencia de otros (Arco Tirado et 
al., 2018; Barriopedro et al., 2018; Duckworth y Quinn, 2009; Karaman et al., 2019), los resultados del análisis factorial confirmatorio no apoyan el uso de la escala como estaba previsto originalmente, aunque es importante aclarar que en dichas investigaciones el ajuste del modelo resultó apenas aceptable y no puede considerarse fuerte. Además, en este estudio, no se encontró un ajuste más satisfactorio en la versión abreviada que en la original, por lo cual no se hallaron motivos para recomendarla como una medida más económica de la perseverancia y la pasión por las metas a largo plazo. Esto evidencia la necesidad de seguir profundizando el estudio de la estructura factorial del instrumento.

\section{Conclusiones}

La presente investigación incrementa el número de estudios que en los últimos años se centraron en aplicar la escala Grit (Duckworth et al., 2007; Duckworth y Quinn, 2009) y efectuar diversos análisis psicométricos en diferentes contextos culturales y lingüísticos, aportando evidencias sobre su validez y ofreciendo un aporte significativo para la evaluación psicopedagógica, psicológica y para el campo de la psicología positiva educacional.

Los resultados obtenidos mostraron que la adaptación de la escala Grit, en ambas versiones original (12 ítems) y abreviada (8 ítems), posee aceptables propiedades psicométricas, semejantes a las halladas en investigaciones similares, y presenta buena validez de constructo, lo que brinda respaldo a su aplicación en población de estudiantes universitarios argentinos e invita a continuar ahondando en el estudio de la estructura factorial del instrumento. Asimismo, permite a los psicopedagogos, psicólogos, educadores e investigadores de este país contar con un instrumento adecuado para evaluar la perseverancia y la pasión en el logro de objetivos a largo plazo, y para correlacionar esta capacidad con otras variables. En efecto, si bien ya existen adaptaciones de las dos versiones en población hispanoparlante, el presente estudio cubre una laguna de conocimiento importante en el contexto en el que se llevó a cabo.

Por otra parte, los análisis para establecer correlaciones entre este instrumento y el compromiso académico evidenciaron resultados similares a los encontrados en otras indagaciones (Datu, et al., 2016; Hodge, et al., 2018; Von Culin, et al., 2014), lo que indica que mayores niveles de Grit se asocian con un elevado compromiso; este efecto, constituiría un predictor positivo de la permanencia y de la obtención de un buen rendimiento académico en los estudiantes universitarios, como así también de su interés por alcanzar metas desafiantes, aspectos que ocupan un lugar preponderante en la agenda educativa del nivel superior; por este mismo motivo, sería un factor que, en el caso de considerarse, ayudaría a contar con información válida y solvente en la tarea de seguimiento del alumnado.

De la misma manera, si se aplicasen los instrumentos utilizados en este estudio en población docente y en orientadores educativos, permitiría disponer de información que enriquezca su seguimiento y brindarles estrategias que faciliten su bienestar laboral, tal como se señala en otro trabajo efectuado en población de habla hispana (Álvarez-Ramírez et al., 2017). Los datos obtenidos a través de las escalas Grit-O y Grit-S en universitarios permitirían generar estrategias, herramientas y modelos predictivos que posibiliten un mejor abordaje y acompañamiento de las trayectorias académicas de los alumnos. Además, pueden ser de sumo valor para orientar su proyecto de vida en los espacios de tutoría o psicopedagógicos, implementando estrategias que faciliten la concreción de metas a largo plazo, a partir de la focalización de objetivos más cercanos en el tiempo. En particular, se destaca la estrategia denominada SMART (Moritz, 2015), que aquí 
se presenta adaptada y cuyo nombre deriva de la primera letra de cinco cualidades que deben condensar los objetivos a corto plazo: 1. Simplicidad, que hace referencia a la calidad de los objetivos (que sean sencillos); 2. Medición, que sean objetivos capaces de ser medidos y evaluados; 3. Amabilidad, que sean objetivos valorados por el alumno; 4. Realidad, que estén vinculados a la vida real del estudiante; 5 . Temporalidad, que estén acotados en el tiempo.

Finalmente, los hallazgos de estudios previos, que evidencian la asociación positiva y el poder predictivo de la autoeficacia académica sobre el Grit en universitarios (Tortul, 2019, noviembre), permiten inferir que al fortalecer en los estudiantes la confianza en sus capacidades para lograr objetivos académicos, especialmente en aquellos que tienen creencias negativas y poco objetivas acerca de su propio rendimiento y su potencial para alcanzar el éxito, se puede incrementar el nivel de Grit en los mismos; es decir, cuanto mayor sea esta creencia, mayores serán las posibilidades de que los alumnos mantengan el interés por su carrera a mediano y largo plazo, que no se desvíen de sus metas, aun cuando tengan que afrontar adversidades y que se esfuercen para afrontar los desafíos y obstáculos que encuentren en este proceso (Duckworth et al., 2007; Duckworth y Quinn, 2009). Por este motivo, será apropiado que profesionales del área psicopedagógica brinden orientación a los docentes de nivel superior para que, sin disminuir las exigencias necesarias para el logro de los objetivos de su espacio de enseñanza, estimulen en el alumnado la motivación y la confianza de que obtendrán resultados positivos, si se esfuerzan.

Entre las limitaciones de esta investigación, debemos mencionar que la muestra fue seleccionada de manera no probabilística, intencional y su composición resultó homogénea, dado que estaba conformada en su totalidad por estudiantes universitarios. En consecuencia, se deben tomar recaudos para la generalización de los resultados y para la aplicación del instrumento a poblaciones con características diferentes.

Asimismo, se deberían realizar estudios en muestras aleatorias y de mayor tamaño a fin de garantizar su representatividad y la generalización de los resultados obtenidos en poblaciones con diversas características con el objeto de verificar si se mantienen las propiedades psicométricas de la escala. Sería interesante también, en futuras investigaciones, indagar las diferencias que existen según el sexo, ya que éstas no fueron analizadas en el presente estudio.

Por otra parte, cabe destacar que se trató de una investigación transversal que podría complementarse con la realización de estudios longitudinales con la finalidad de observar la evolución del constructo en el transcurso de determinada cantidad de tiempo.

En futuras investigaciones deberían extenderse y profundizarse los análisis de las propiedades psicométricas de la adaptación argentina de la escala Grit, como así también de su asociación con otras variables.

\section{Referencias bibliográficas}

Alvarez-Garrido, Ma de los R., Pena Garrido, M. y Losada Vicente, L. (2017). Misión posible: mejorar el bienestar de los orientadores a través de su inteligencia emocional. Revista

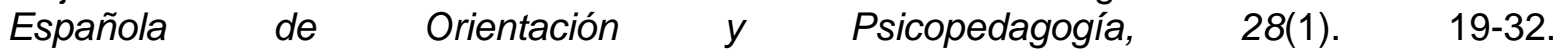
https://doi.org/10.5944/reop.vol.28.num.1.2017.19356 
Arco-Tirado, J. L., Fernández-Martín, F. D. y Hoyle, R. H. (2018). Development and validation of a Spanish version of the Grit-S scale. Frontiers in Psychology, 9, 96. https://doi.org/10.3389/fpsyg.2018.00096

Akin, A., Abaci, R., Arici, N., Uysal, R. y Uysal, Ç. K. (2011, 8 de septiembre). Revised Grit Scale: The validity and reliability study. En XX National Education Symposium (Burdur, Turquía).

Barni, M.C. y Daura, F.T. (2019). Is Grit the Key Element to Improve the Life Attitude? A Study with Military Students from Argentina. European Journal of Social Sciences, 2(3). 30-41. http://dx.doi.org/10.26417/ejss.v2i3.p30-41

Barriopedro, M. I., Quintana, I. y Ruiz, L. M. (2018). La perseverancia y pasión en la consecución de objetivos: validación española de la Escala Grit de Duckworth. RICYDE. Revista Internacional de Ciencias del Deporte. 54(14), 297-308. https://doi.org/10.5232/ricyde2018.05401

Becerra Martín, D., Cuitún Coronado, J. y Mézquita Hoyos, Y. (2016, 7 de septiembre). Validación de la Escala de tenacidad en estudiantes oaxaqueños: un estudio exploratorio. En XVI Congreso Mexicano de Psicología Social, en el VII Congreso Internacional de Relaciones Personales y en el V Congreso Latinoamericano de Psicología Transcultural, Tabasco.

Bentler, P. M. (1990). Comparative fit indexes in structural models. Psychological Bulletin, 107(2), 238. https://psycnet.apa.org/doi/10.1037/0033-2909.107.2.238

Cambridge University Press (2018). Cambridge Dictionary. Cambridge University Press. https://dictionary.cambridge.org/es/diccionario/ingles/grit

Credé, M., Tynan, M. C. y Harms, P. D. (2017). Much ado about grit: A meta-analytic synthesis of the grit literature. Journal of Personality and Social Psychology, 113(3), 492-511. https://doi.org/10.1037/pspp0000102

Datu, J.A.D., Valdez, J.P.M. y King, R.B. (2016). The Successful Life of Gritty Students: Grit Leads to Optimal Educational and Well-Being Outcomes in a Collectivist Context. En R. King y A. Bernardo (Eds). The Psychology of Asian Learners. Springer.

Daura, F.T., Barni, Maㅡ C., González, M., Assirio, J. y Lúquez, G. (2019, agosto). Temples de acero, comprometidas. Una aproximación al estudio del compromiso académico y del grit en estudiantes de postgrados orientados al desarrollo del liderazgo. En XVII reunión Nacional y VI Encuentro Internacional de la Asociación Argentina de Ciencias del Comportamiento (Posadas, Argentina).

Duckworth, A. L. (2013, 17 de abril). Grit: la clave del éxito. TED Talks Education [video]. YouTube. https://goo.gl/RgCZ6r

Duckworth, A. L. (2016). Grit: The power of passion and perseverance. Scribner.

Duckworth, A. L., Kirby, T., Tsukayama, E., Berstein, H. y Ericsson, K. A. (2010). Deliberate practice spells success: Why grittier competitors triumph at the National Spelling Bee. Social Psychological and Personality Science, 2, 174-181. https://doi.org/10.1177/1948550610385872

Duckworth, A. L., Peterson, Ch., Matthews, M. y Kelly, D. (2007). Grit: Perseverance and Passion for Long-Term Goals. Journal of Personality and Social Psychology, 92(6), 1087-1101. https://doi.org/10.1037/0022-3514.92.6.1087

Duckworth, A. L. y Quinn, P. D. (2009). Development and validation of the Short Grit Scale (GRITS). Journal of personality assessment, 91(2), 166-174. https://doi.org/10.1080/00223890802634290 
Duckworth, A.L., Quinn, P.D. y Seligman, M.E.P. (2009). Positive predictors of teacher effectiveness. The Journal of Positive Psychology: Dedicated to furthering research and promoting good practice, 4(6), 540-547. https://doi.org/10.1080/17439760903157232

Eskreis-Winkler, L., Duckworth, A. L., Shulman, E. y Beal, S. (2014). The grit effect: Predicting retention in the military, the workplace, school, and marriage. Frontiers in Psychology, 5(36), 1-12. https://doi.org/10.3389/fpsyg.2014.00036

Fernández-Martín, F. D., Arco-Tirado, J. L. y Soriano-Ruíz, M. (2018). Perseverance and passion for achieving long-term goals: transcultural adaptation and validation of the Grit-S scale/Perseverancia y pasión por la consecución de objetivos a largo plazo: adaptación transcultural y validación de la escala Grit-S. Revista de Psicología Social, 33(3), 620-649. https://doi.org/10.1080/02134748.2018.1482060

Ferrando, P. J. y Lorenzo-Seva U. (2016). A note on improving EAP trait estimation in oblique factor-analytic and item response theory models. Psicológica, 37, 235-247. https://www.redalyc.org/pdf/169/16946248007.pdf

Hodge, B., Wrigth, B. y Bennet, P. (2018). The Role of Grit in Determining Engagement and Academic Outcomes for University Students. Research in Higher Education, 59(4), 448460. https://doi.org/10.1007/s11162-017-9474-y

Hu, L. T. y Bentler, P.M. (1998). Fit indices in covariance structure modeling: Sensitivity to under parameterized model misspecification. Psychological Methods, 3(4), 424-453. https://psycnet.apa.org/doi/10.1037/1082-989X.3.4.424

International Tests Commission (2001). International guidelines for test use. International Journal of Testing, 1(2), 93-114. https://doi.org/10.1207/S15327574IJT0102_1

Karaman, M. A., Vela, J. C., Aguilar, A. A., Saldana, K. y Montenegro, M. C. (2019). Psychometric properties of US-Spanish versions of the grit and resilience scales with a Latinx population. International Journal for the Advancement of Counselling, 41(1), 125-136. https://doi.org/10.1007/s10447-018-9350-2

Kline, R. B. (2005). Principles and practice of structural equation modeling (2nd. Ed.). Guilford.

Lorenzo-Seva, U. y Ferrando, P.J. (2020). Manual of the program Factor. http://psico.fcep.urv.es/utilitats/factor/index.html

Lloret-Segura, S., Ferreres-Traver, A., Hernandez-Baeza, A. y Tomas-Marco, I. (2014). El Análisis factorial exploratorio de los ítems: una guía práctica, revisada y actualizada. Anales de Psicología, 30(3), 1151-1169. https://doi.org/10.6018/analesps.30.3.199361.

Mesurado, B., Richaud, M. C. y Mateo, N. J. (2016). Engagement, Flow, Self-Efficacy, and Eustress of University Students: A Cross-National Comparison Between the Philippines and Argentina. The Journal of Psychology, 150(3), 281-299. https://doi.org/10.1080/00223980.2015.1024595

Montero, I. y León, O. (2007). A guide for naming research studies in Psychology. International Journal of Clinical and Health Psychology. 7, 847-862. https://www.redalyc.org/articulo.oa?id=337/33770318

Moritz, A. (2015, 25 de noviembre). Building Students Skills that lead to resilience and academic tenacity. Center for Schools and Communities. [presentación de diapositivas]. https://asdn.org/wp-content/uploads/Grit-PPT-Handout-1.pdf 
Muñiz, J., Elosua, P. y Hambleton, R. K. (2013). Directrices para la traducción y adaptación de los tests: segunda edición. Psicothema, 25, 151-157. https://doi.org/10.7334/psicothema2013.24

Nishikawa, K., Okugami, S. y Amemiya, T. (2015). Development of the Japanese Short Grit Scale (Grit-S). The Japanese Journal of Personality, 24, 167-169. https://doi.org/10.2132/personality.24.167

Perkins-Gough, D. (2013). The Significance of Grit: A Conversation with Angela Lee Duckworth. Educational Leadership, 71(1), 14-20.

Robertson-Kraft, C. y Duckworth, A. L. (2014). True Grit: Trait-level Perseverance and Passion for Long-term Goals Predicts Effectiveness and Retention among Novice Teachers. Teachers College Record (1970), 116(3), http://www.tcrecord.org/Content.asp?Contentld=17352.

Schaufeli, W. B., Martínez, I. M., Marques Pinto, A., Salanova, M. y Bakker, A. B. (2002). Burnout and engagement in university students: A cross-national study. Journal of Cross-Cultural Psychology, 33, 464-481. https://doi.org/10.1177/0022022102033005003

Schaufeli, W. B., Salanova, M., González-Romá, V. y Bakker, A. B. (2002). The measurement of engagement and burnout: A two sample confirmatory factor analytic approach. Journal of Happiness Studies, 3(1), 71-92. https://doi.org/10.1023/A:1015630930326

Suzuki, Y., Tamesue, D., Asahi, K. y Ishikawa, Y. (2015). Grit and Work Engagement: A CrossSectional Study. PloS one, 10(9), e0137501, https://doi.org/10.1371/journal.pone.0137501

Tomarken, A. J. y Waller, N. G. (2005). Structural equation modeling: Strengths, limitations, and misconceptions. Annual Review of Clinical Psychology, 1, 31-65. https://doi.org/10.1146/annurev.clinpsy.1.102803.144239

Tortul, M. C. (2019, 27 de noviembre). Influencia de la autoeficacia en la perseverancia y la pasión por alcanzar metas a largo plazo (GRIT) en estudiantes universitarios de Paraná, Entre Ríos. En XI Congreso Internacional de Investigación y Práctica Profesional en Psicología. XXVI Jornadas de Investigación. XV Encuentro de Investigadores en Psicología del MERCOSUR. Facultad de Psicología-UBA (Buenos Aires, Argentina). https://www.aacademica.org/000-111/823

Von Culin, K. R., Tsukayama, E. y Duckworth, A. L. (2014) Unpacking grit: Motivational correlates of perseverance and passion for long-term goals. The Journal of Positive Psychology, 9(4), 306-312, https://doi.org/10.1080/17439760.2014.898320

Yeager, D. S., Henderson, M. D., Paunesku, D., Walton, G. M., D'Mello, S., Spitzer, B. J. y Duckworth, A. L. (2014). Boring but important: a self-transcendent purpose for learning fosters academic self-regulation. Journal of Personality and Social Psychology, 107(4), 559-80. https://doi.org/10.1037/a0037637.

Fecha de entrada: 1 julio de 2019

Fecha de revisión: 21 abril de 2020

Fecha de aceptación: 13 agosto 2020 